\title{
Various species of Pyricularia constitute a robust clade distinct from Magnaporthe salvinii and its relatives in Magnaporthaceae
}

\author{
Nobuaki Murata • Takayuki Aoki • Motoaki Kusaba • \\ Yukio Tosa $\cdot$ Izumi Chuma
}

Received: 19 March 2013/Accepted: 28 June 2013/Published online: 4 September 2013

(C) The Phytopathological Society of Japan and Springer Japan 2013

\begin{abstract}
In a phylogenetic analysis of species of Magnaporthaceae based on nucleotide sequences of rDNA-ITS and the RPBI gene, isolates of the tested species were divided into two clusters with high bootstrap support. One group was composed of Pyricularia spp.; the other was composed of Magnaporthe salvinii, M. rhizophila, M. poae, Gaeumannomyces graminis, and G. incrustans. On the basis of this result, we concluded that Pyricularia spp. constitute a large but distinct phylogenetic species group that is not congeneric with Magnaporthe salvinii, the type species of Magnaporthe.
\end{abstract}

\section{Keywords Magnaporthe Pyricularia .}

Gaeumannomyces

The generic name, Pyricularia, has been assigned to the anamorphs of filamentous fungi that cause blast disease on monocot species. The best known species is $P$. oryzae Cavara, pathogenic on staple crops including rice, wheat,

Electronic supplementary material The online version of this article (doi:10.1007/s10327-013-0477-z) contains supplementary material, which is available to authorized users.

N. Murata $\cdot$ Y. Tosa $\cdot$ I. Chuma $(\bowtie)$

Laboratory of Plant Pathology, Graduate School of Agricultural

Sciences, Kobe University, Kobe 657-8501, Japan

e-mail: chuizm@kobe-u.ac.jp

T. Aoki

Genetic Resources Center, National Institute of Agrobiological

Sciences, Tsukuba 305-8602, Japan

M. Kusaba

Laboratory of Plant Pathology, Faculty of Agriculture, Saga

University, Saga 840-8502, Japan and millet (Kato et al. 2000). Among its close relatives are P. grisea Saccardo, pathogenic on crabgrass, Pyricularia sp. (LS) isolated from Leersia/Setaria, and Pyricularia sp. (CE) isolated from Cenchrus/Echinochloa (Hirata et al. 2007). These four species are members of the P. oryzael grisea species complex and indistinguishable in conidial morphology. Pyricularia also includes several species defined by morphological features such as $P$. zizaniaecola Hashioka, pathogenic on Zizania, P. zingiberi Y. Nisikado on Zingiber, P. higginsii Luttr. on Cyperus, Pyricularia sp. (SsPb) on Sasa/Phyllostachys (Hirata et al. 2007).

In the $1970 \mathrm{~s}$, teleomorphs of $P$. grisea and $P$. oryzae were discovered in several laboratories (Hebert 1971; Kato et al. 1976; Ueyama and Tsuda 1975; Yaegashi and Nishihara 1976). These species produced nonstromatic black perithecia with long necks and four-celled, spindleshaped ascospores. The teleomorphs of $P$. grisea and $P$. oryzae were, at first, collectively designated as Magnaporthe grisea (T.T. Hebert) M.E. Barr (Barr 1977; Yaegashi and Udagawa 1978), but they are now called M. grisea and M. oryzae B.C. Couch, respectively (Couch and Kohn 2002).

The blast fungi are primarily airborne and colonize leaves and panicles of host plants. However, the genus Magnaporthe also includes soilborne, root-infecting species such as M. rhizophila D.B. Scott \& Deacon (Scott and Deacon 1983), and M. poae Landschoot \& N. Jackson (Landschoot and Jackson 1989). In addition, the family Magnaporthaceae (Cannon 1994), typified by the genus Magnaporthe, includes Gaeumannomyces spp., widely distributed soilborne pathogens. These two groups (airborne and soilborne species) also differ in their anamorphs; the blast fungi produce pyriform conidia, whereas $M$. rhizophila, M. poae, and Gaeumannomyces spp. produce Phialophora-like conidia (Zhang et al. 2011). Furthermore, 


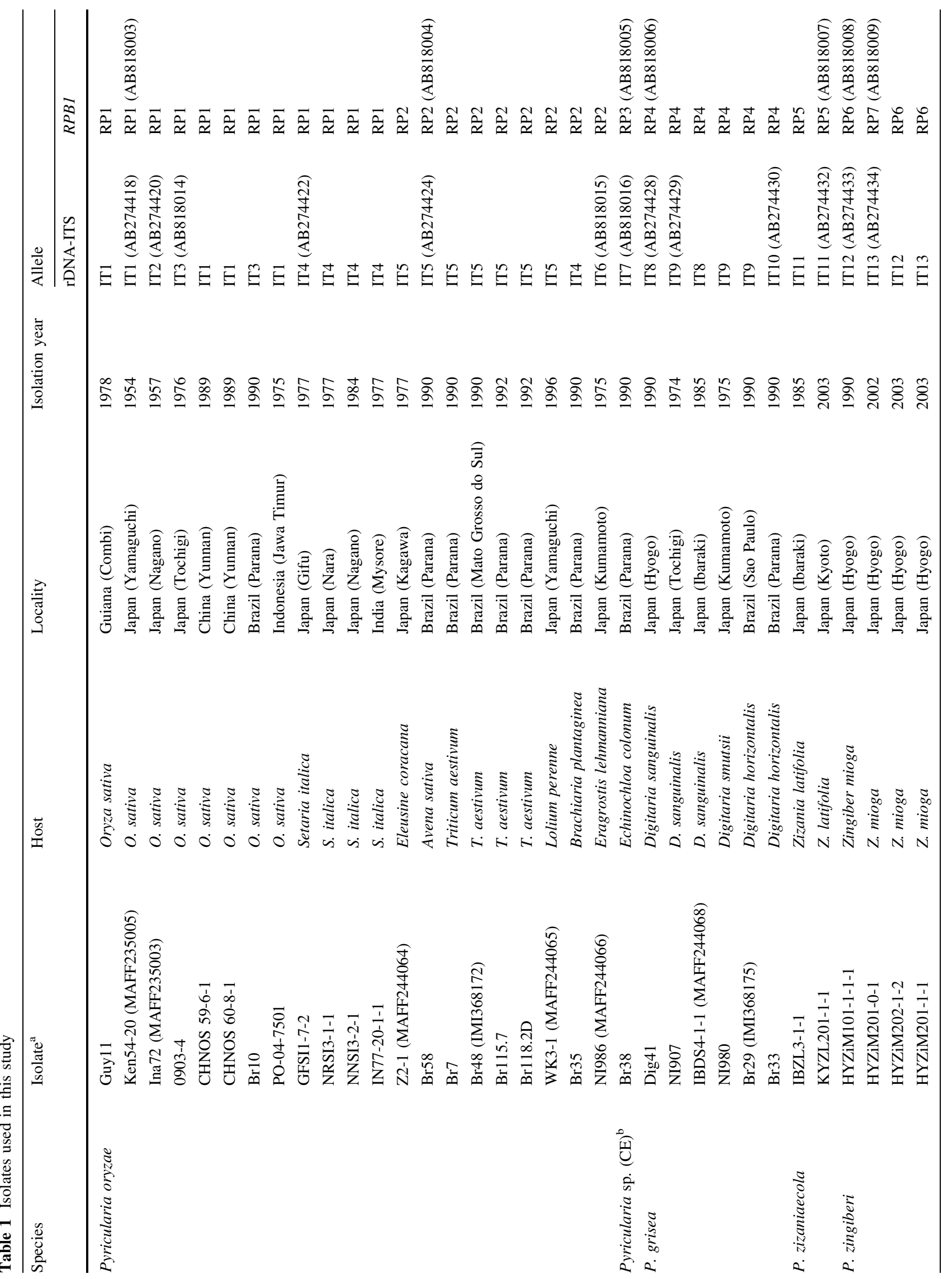




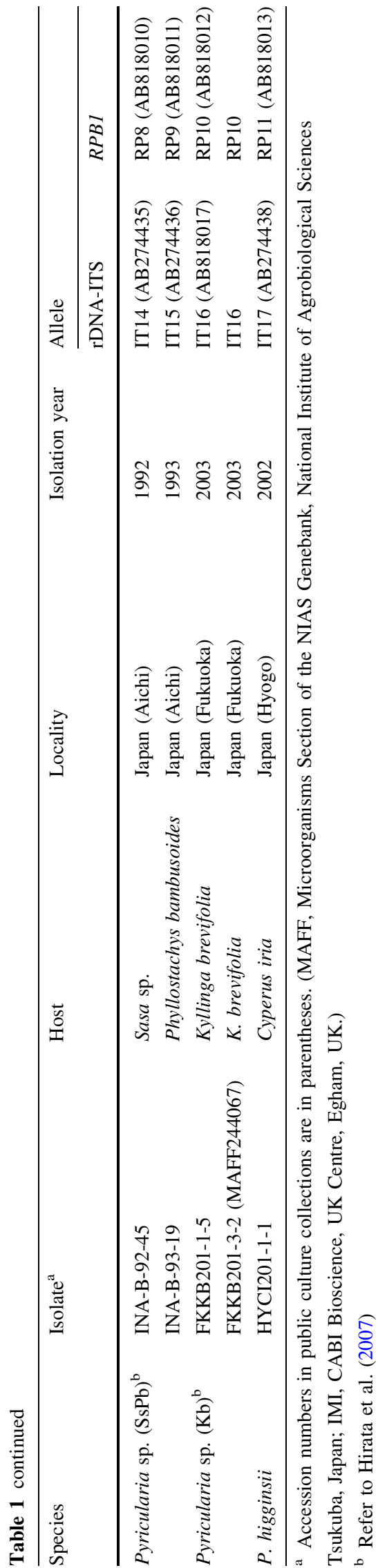

M. salvinii (Catt.) R.A. Krause \& R.K. Webster, the type species of the genus (Krause and Webster 1972), is different from both of the two groups; its infection cycle is primarily dependent on sclerotia that colonize the leaf sheath although it forms bicolored conidia. This circumstantial evidence led Zhang et al. (2011) to question whether Magnaporthe and Gaeumannomyces were monophyletic taxa. On the basis of the results from multilocus phylogenetic analyses, they considered that both Magnaporthe and Gaeumannomyces were polyphyletic and suggested that anamorphic and ecological features were more informative than the teleomorphic characters in defining monophyletic natural groups. However, they used only four M. oryzae/P. oryzae isolates as representatives of Pyricularia for their analysis. As mentioned already, several phylogenetically distinct, morphological species (Hirata et al. 2007) have been described in Pyricularia. Their teleomorphs have not yet been discovered, and their phylogenetic positions in Magnaporthaceae have been still unclear.

At the nomenclatural sessions of the International Botanical Congress in Melbourne, 2011, it was decided that, after 1 January 2013, one fungus can only have one correct name (Hawksworth 2011) and that other names will be considered synonyms. Based on the new nomenclatural code for algae, fungi, and plants (McNeill et al. 2012), all legitimate fungal names are treated equally for the purposes of establishing priority (Wingfield et al. 2012). Therefore, $P$. oryzae and $P$. grisea as former anamorphic names may compete with former teleomorphic names $M$. oryzae and $M$. grisea, respectively, for priority. In the present study, we performed phylogenetic analyses using diverse blast fungi including various species. As a result of the obtained phylogenetic structure of Magnaporthaceae, we discuss which generic name should be adopted for the blast fungi, Magnaporthe or Pyricularia.

The Pyricularia isolates tested are listed in Table 1. Genomic DNA was extracted as described by Nakayashiki et al. (1999). According to the number of informative sites reported by Zhang et al. (2011), two loci were selected for analysis: a portion of the nu-rRNA gene repeat (rDNA-ITS: ITS1, 5.8S and ITS2) and a portion of the largest subunit of RNA polymerase II gene (RPBI). The rDNA-ITS region was amplified with primers ITS5 (5'-GGAAGTAAAAG TCGTAACAAGG- $\left.3^{\prime}\right)$ and ITS4 (5'-TCCTCCGCTTATT GATATGC-3') (White et al. 1990) as described by Hirata et al. (2007). The RPBI gene was amplified in a $20 \mu \mathrm{L}$ reaction containing $1 \mathrm{U}$ rTaq DNA polymerase (TOYOBO, Osaka, Japan), $1 \times$ PCR buffer provided by the manufacturer, $200 \mu \mathrm{M}$ each dNTP, $0.2 \mu \mathrm{M}$ primers RPB1-Ac $\left(5^{\prime}-\mathrm{G}\right.$ ARTGYCCDGGDCAYTTYGG-3') and RPB1-Cr (5'-CC NGCDATNTCRTTRTCCATRTA-3') (Zhang et al. 2011), $1.5 \mathrm{mM} \mathrm{MgCl}_{2}$, and $1 \mathrm{ng}$ of template DNA using a 


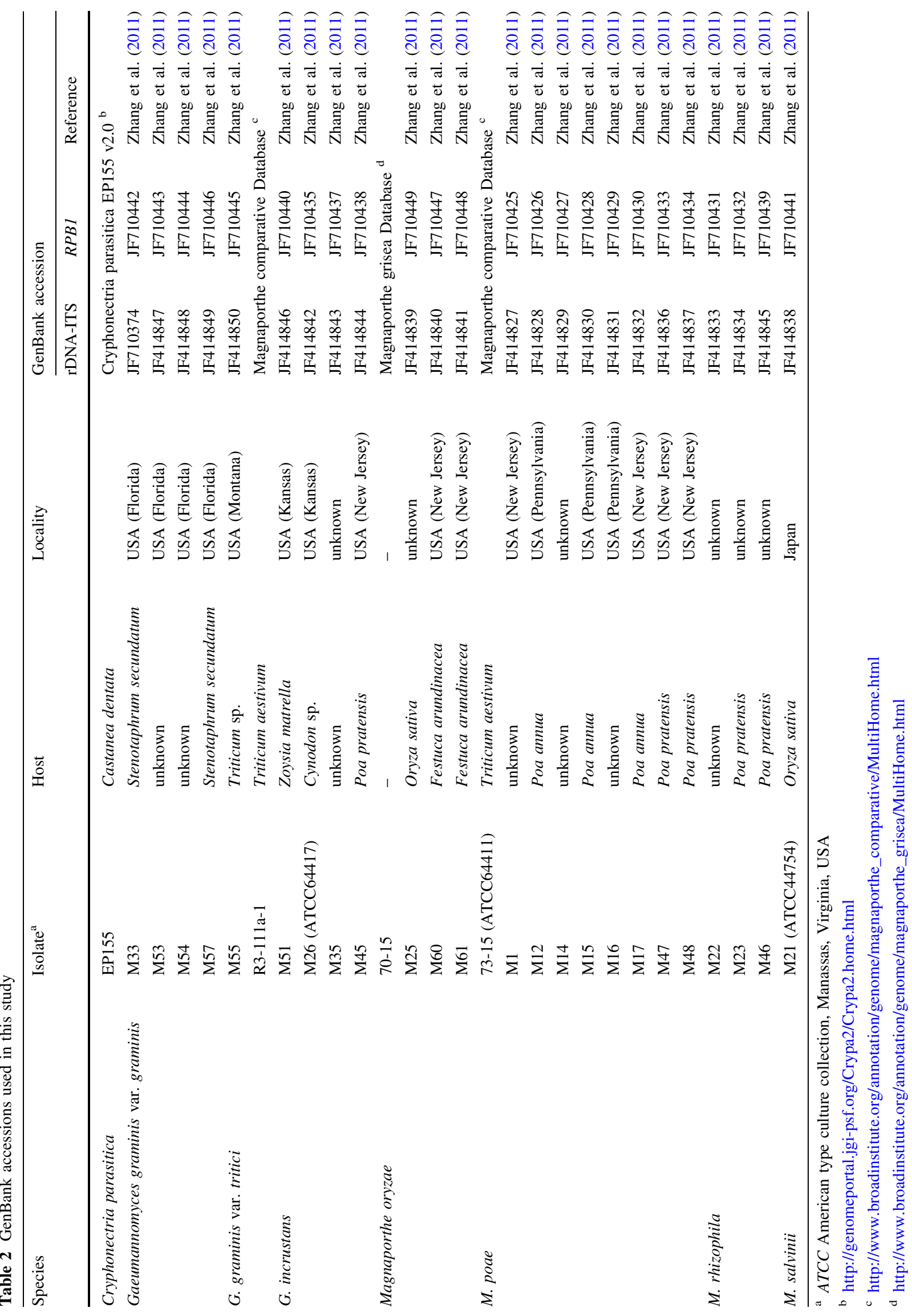


$M L$ tree
P. oryzae (M. oryzae) 70-15 MG6

$P$. oryzae (M. oryzae) Guy11

P. oryzae (M. oryzae) Ken54-20

88

P. oryzae (M. oryzae) CHNOS 59-6-1

P. oryzae (M. oryzae) CHNOS 60-8-1

P. oryzae (M. oryzae) PO-04-7501

$P$. oryzae (M. oryzae) Ina72

$P$ oryzae (M oryzae) $0903-4$

P. oryzae (M. oryzae) Br10

$P$. oryzae (M. oryzae) GFSI1-7-2

P. oryzae (M. oryzae) NRSI3-1-1

P. oryzae (M. oryzae) NNSI3-2-1

$P$. oryzae (M. oryzae) IN77-20-1-1

$P$. oryzae (M. oryzae) M25

P. oryzae (M. oryzae) M60

100

P. oryzae (M. oryzae) Z2-1

$P$. oryzae (M. oryzae) Br58

$P$. oryzae (M. oryzae) Br7

P. oryzae (M. oryzae) Br48

P. oryzae (M. oryzae) Br118.2D

P. oryzae (M. oryzae) WK3-1

P. oryzae (M. oryzae) $\mathrm{Br} 35$

P. oryzae (M. oryzae) NI986

100

.

Pyricularia sp. (CE) Br38

$P$. grisea (M. grisea) Dig41

$P$ grisea ( $M$. grisea) IBDS4-1-1

$P$. grisea (M. grisea) N1980

P. grisea (M. grisea) Br29

$P$. grisea (M. grisea) NI907

P. grisea (M. grisea) $\mathrm{Br} 33$

100 P. zizaniaecola IBZL3-1-1

P. zizaniaecola KYZL201-1-1

P. zingiberi HYZiM101-1-1-1

P. zingiberi HYZiM202-1-2

P. zingiberi HYZiM201-1-1

P. zingiberi HYZiM201-0-1

100

100 Pyricularia sp. (Kb) FKKB201-1-5

Pyricularia sp. (Kb) FKKB201-3-2

P. higginsii $\mathrm{HYCl} 201-1-1$

Pyricularia sp. (SsPb) INA-B-92-45

100 Pyricularia sp. (SsPb) INA-B-93-19

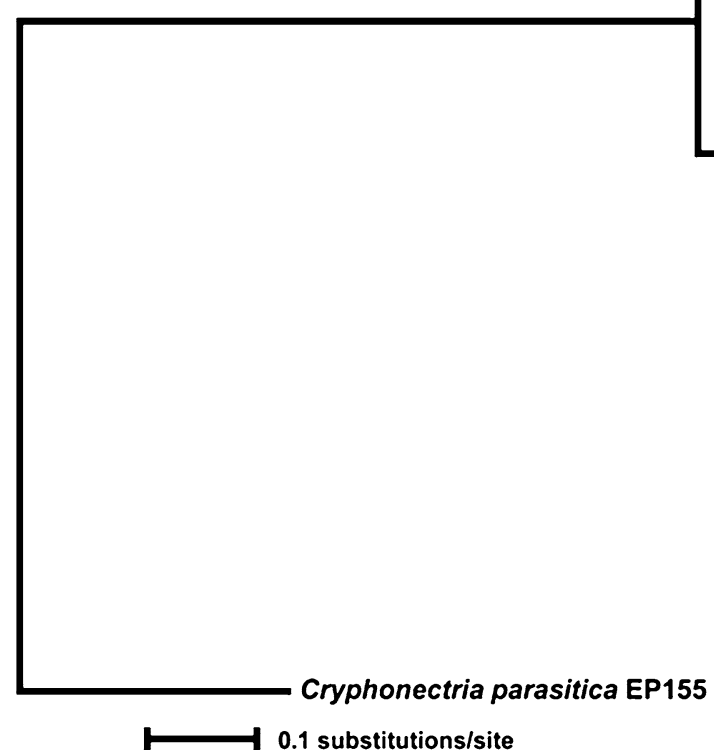

Fig. 1 Maximum likelihood (ML) phylogenetic tree based on rDNAITS (ITS1, 5.8S and ITS2) and RPB1 nucleotide sequences of Magnaporthaceae isolates. The tree was rooted using Cryphonectria

Mastercycler (Eppendorf, Hamburg, Germany). PCR cycling conditions for $R P B 1$ were $1 \mathrm{~min}$ at $95^{\circ} \mathrm{C} ; 30$ cycles of $1 \mathrm{~min}$ at $94{ }^{\circ} \mathrm{C}, 1 \mathrm{~min}$ at $60{ }^{\circ} \mathrm{C}, 1 \mathrm{~min}$ at $72{ }^{\circ} \mathrm{C}$;

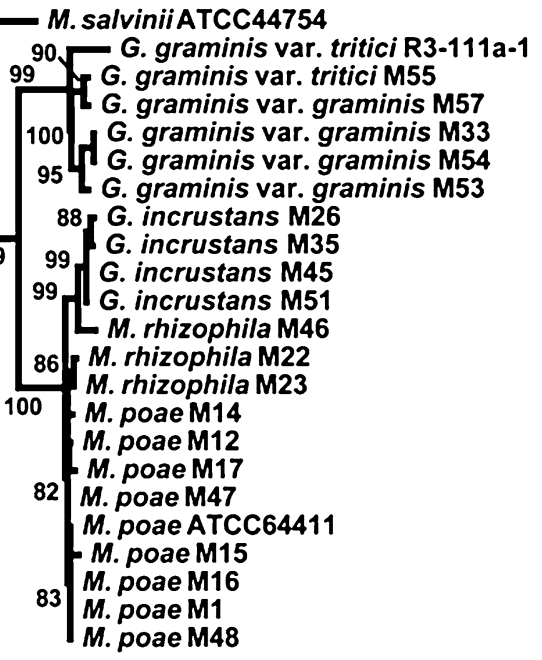

parasitica EP155 as an outgroup. Numbers at nodes represent bootstrap support $>75 \%$ from 500 replicates

and 5 min at $72{ }^{\circ} \mathrm{C}$. PCR products were purified with USB ExoSAP-IT (Affymetrix, Santa Clara, CA, USA), and sequenced directly with the same primers as in the 
amplification using BigDye Terminator v3.1 Cycle Sequencing Kit and ABI PRISM 3130xl Genetic Analyzer (Life Technologies, Carlsbad, CA, USA). The sequences were assembled with SeqMan II (DNASTAR, Madison, WI, USA) and deposited in GenBank (see Table 1). The nucleotide sequences of rDNA-ITS and RPB1 reported by Zhang et al. (2011) and those of model organisms (Cryphonectria parasitica EP155, Gaeumannomyces graminis var. tritici R3-111a-1, Magnaporthe oryzae 70-15 and M. poae ATCC 64411) were downloaded from GenBank and genome databases (Table 2).

Nucleotide sequences of each locus were aligned with the program CLUSTAL W (Thompson et al. 1994), and manually optimized using the program MEGA 5.10 (Tamura et al. 2011). Combined alignments were analyzed using maximum parsimony (MP), maximum likelihood (ML), and Bayesian inference (BI) methods. Cryphonectria parasitica EP155 was used as an outgroup. The MP analysis was performed with MEGA 5.10 using the CloseNeighbor-Interchange (CNI) algorithm at a search level of 1. The initial tree for the CNI search was created by random addition for 10 replications. Nodal supports were assessed using 500 bootstrap replicates. The best-fit model for the ML and BI analyses was selected using the corrected Akaike information criteria in the program jModelTest 2.1.1 (Darriba et al. 2012; Guindon and Gascuel 2003). The ML analysis with the GTR $+\mathrm{G}$ model was carried out using MEGA 5.10. Nodal supports were assessed using 500 bootstrap replicates. Bayesian analysis was conducted with the program MrBayes 3.2.1 (Ronquist et al. 2012) using the GTR + G model and consisted of two runs of four chains each. The two runs were performed for 500,000 generations, sampling every 100 generations. Average standard deviations of split frequency values lower than 0.01 were taken as an indication that convergence had been achieved. After the first 1,250 trees were discarded, a $50 \%$ majority rule consensus tree was constructed based on the remaining samples. The tree was visualized using the program FigTree v1.4.0 (available at http://tree.bio.ed.ac.uk/software).

The ML tree is shown in Fig. 1. The isolates of Magnaporthaceae species tested were divided into two clusters with high bootstrap support. One cluster was composed of the blast fungi and harbored all of the morphologically distinct Pyricularia species (Fig. 2). The other was composed of soilborne pathogens, M. rhizophila, M. poae, G. graminis, and G. incrustans. Magnaporthe salvinii was included in the latter cluster. Gaeumannomyces was split into two subclusters; one was composed of all isolates of G. graminis var. graminis and G. graminis var. tritici, while the other was $G$. incrustans, grouped together with soilborne Magnaporthe species. Similar results were obtained from the MP and BI analyses (Online resources 1 and 2).

As mentioned already, the species analyzed in the present study is divided into three groups based on the ecological and anamorphic features; (1) Pyricularia spp. mainly colonizing leaves and panicles, (2) Gaeumannomyces spp. and soilborne Magnaporthe spp. colonizing roots, and (3) M. salvinii colonizing leaf sheath. The present study showed that M. salvinii, the type species of Magnaporthe, is clustered with group (2) and distinct from group (1) (the blast fungi). The high bootstrap support at the nodes of the two clusters suggests that each of them is a monophyletic clade derived from a common ancestor. Although species classified in the same genus Magnaporthe are found in both clades, their differences in anamorphs and infection behaviors are not so trivial as those found among species of the same genus. We suggest that the two clades should logically be separated as distinct genera and have different generic names.

Tsuda and Ueyama (1982), one of the three teams that discovered the teleomorph of $P$. oryzae (Ueyama and Tsuda 1975), found that the teleomorph of the blast fungus was different from typical Magnaporthe in morphology of ascospores, especially structure at their tips, and mode of their germination. Based on these observations, they concluded that the teleomorph of the blast fungus should not
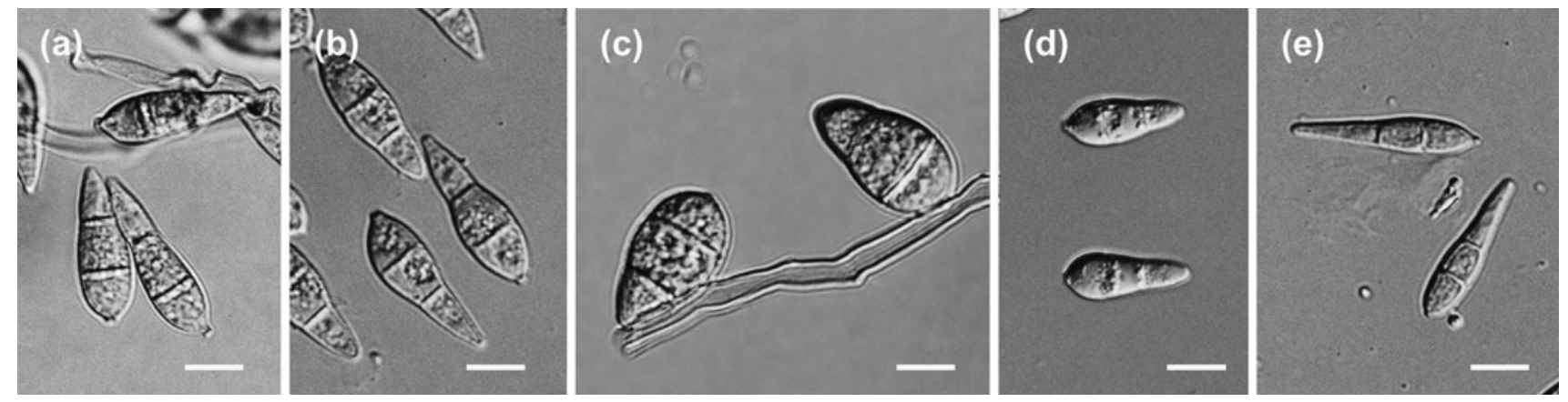

Fig. 2 Pyriform conidia produced by Pyricularia spp. a $P$. oryzae (Triticum isolate, Br48). b $P$. grisea (Digitaria isolate, Dig41). c $P$. zizaniaecola (Zizania isolate, KYZL201-1-1). d P. zingiberi (Zingiber isolate, TKZiM202-1). e P. higginsii (Cyperus isolate, HYCI201-1-1). Bars $=10 \mu \mathrm{m}$ 
be designated as Magnaporthe and argued that a new teleomorph genus should be established for it. Our phylogenetic analyses also suggest that Magnaporthe, typified by M. salvinii, should not be connected with the blast fungi, that have long been called Pyricularia species.

Luo and Zhang (2013) suggested synonymizing M. salvinii to Nakataea oryzae (Cattaneo) J. Luo \& Zhang, recombined from Sclerotium oryzae Cattaneo, an anamorphic synonym of $M$. salvinii. According to this treatment, the generic name Magnaporthe will correspondently be synonymized to Nakataea Hara. It should be noted that Nakataea is apparently different from Pyricularia, especially in conidial shape and pigmentation (Luo and Zhang 2013). Based on their morphological and phylogenetic considerations, they suggested that the blast fungus should not be congeneric with the type species of Magnaporthe.

Taken together, we conclude that the blast fungi composed of various species constitute a large, but distinct phylogenetic group of fungi that is not congeneric with M. salvinii, the type species of Magnaporthe. If Magnaporthe is adopted as the generic name of the blast fungi, therefore, the type species of Magnaporthe must be replaced with some species in the clade of blast fungi. On the basis of these considerations, we propose that the clade of the blast fungi should be designated Pyricularia simply based on its priority, as suggested by Luo and Zhang (2013), and that the name Magnaporthe should be used for its type or closely related species in the M. salvinii clade.

Acknowledgments We thank Dr. H. Kato, former professor of Kobe University, for providing the isolates and valuable suggestions. We also thank Dr. B. Valent, Kansas State University, for critical reading the manuscript and valuable suggestions and Dr. N. Zhang, Rutgers University for helpful comments and information that improved the manuscript.

\section{References}

Barr ME (1977) Magnaporthe, Telimenella, and Hyponectria (Physosporellaceae). Mycologia 69:952-966

Cannon PF (1994) The newly recognized family Magnaporthaceae and its interrelationships. Syst Ascomycet 13:25-42

Couch BC, Kohn LM (2002) A multilocus gene genealogy concordant with host preference indicates segregation of a new species, Magnaporthe oryzae, from M. grisea. Mycologia 94:683-693

Darriba D, Taboada GL, Doallo R, Posada D (2012) jModelTest 2: more models, new heuristics and parallel computing. Nat Methods 9:772

Guindon S, Gascuel O (2003) A simple, fast and accurate algorithm to estimate large phylogenies by maximum likelihood. Syst Biol 52:696-704

Hawksworth DL (2011) A new dawn for the naming of fungi: impacts of decisions made in Melbourne in July 2011 on the future publication and regulation of fungal names. IMA Fungus $2: 155-162$

Hebert TT (1971) The perfect stage of Pyricularia grisea. Phytopathology 61:83-87
Hirata K, Kusaba M, Chuma I, Osue J, Nakayashiki H, Mayama S, Tosa Y (2007) Speciation in Pyricularia inferred from multilocus phylogenetic analysis. Mycol Res 111:799-808

Kato H, Yamaguchi T, Nishihara N (1976) The perfect state of Pyricularia oryzae Cav. in culture. Ann Phytopath Soc Jpn 42:507-510

Kato H, Yamamoto M, Yamaguchi-Ozaki T, Kadouchi H, Iwamoto Y, Nakayashiki H, Tosa Y, Mayama S, Mori N (2000) Pathogenicity, mating ability and DNA restriction fragment length polymorphisms of Pyricularia populations isolated from Gramineae, Bambusideae and Zingiberaceae plants. J Gen Plant Pathol 66:30-47

Krause RA, Webster RK (1972) The morphology, taxonomy, and sexuality of the rice stem rot fungus, Magnaporthe salvinii (Leptosphaeria salvinii). Mycologia 64:103-114

Landschoot PJ, Jackson N (1989) Magnaporthe poae sp. nov., a hyphopodiate fungus with a Phialophora anamorph from grass roots in the United States. Mycol Res 93:59-62

Luo J, Zhang N (2013) Magnaporthiopsis, a new genus in Magnaporthaceae (Ascomycota). Mycologia 105:1019-1029

McNeill J, Barrie FR, Buck WR, Demoulin V, Greuter W, Hawksworth DL, Herendeen PS, Knapp S, Marhold K, Prado J, Prud'homme van Reine WF, Smith GF, Wiersema JH, Turland NJ (eds) (2012) International Code of Nomenclature for algae, fungi, and plants (Melbourne Code) adopted by the Eighteenth International Botanical Congress Melbourne, Australia, July 2011. Koeltz Scientific Books, Koenigstein

Nakayashiki H, Kiyotomi K, Tosa Y, Mayama S (1999) Transposition of the retrotransposon MAGGY in heterologous species of filamentous fungi. Genetics 153:693-703

Ronquist F, Teslenko M, van der Mark P, Ayres DL, Darling A, Höhna S, Larget B, Liu L, Suchard MA, Huelsenbeck JP (2012) MrBayes 3.2: efficient Bayesian phylogenetic inference and model choice across a large model space. Syst Biol 61:539-542

Scott DB, Deacon JW (1983) Magnaporthe rhizophila sp.nov., a dark mycelial fungus with a Phialophora conidial state, from cereal roots in South Africa. Trans Br Mycol Soc 81:77-81

Tamura K, Peterson D, Peterson N, Stecher G, Nei M, Kumar S (2011) MEGA5: molecular evolutionary genetics analysis using maximum likelihood, evolutionary distance, and maximum parsimony methods. Mol Biol Evol 28:2731-2739

Thompson JD, Higgins DG, Gibson TJ (1994) CLUSTAL W: improving the sensitivity of progressive multiple sequence alignment through sequence weighting, positions-specific gap penalties and weight matrix choice. Nucleic Acids Res 22:4673-4680

Tsuda M, Ueyama A (1982) A comment from a taxonomical viewpoint on the perfect state of the blast fungus. Ann Phytopathol Soc Jpn (abstract in Japanese) 48:340

Ueyama A, Tsuda M (1975) Formation of the perfect state in culture of Pyricularia sp. from some gramineous plants (preliminary report). Trans Mycol Soc Jpn 16:420-422

White TJ, Bruns T, Lee S, Taylor J (1990) Amplification and direct sequencing of fungal ribosomal RNA genes for phylogenetics. In: Innis MA, Gelfand DH, Sninsky JJ, White TJ (eds) PCR protocols: a guide to methods and applications. Academic Press, San Diego, pp 315-322

Wingfield MJ, De Beer ZW, Slippers B, Wingfield BD, Groenewald JZ, Lombard L, Crous PW (2012) One fungus, one name promotes progressive plant pathology. Mol Plant Pathol 13:604-613

Yaegashi H, Nishihara N (1976) Production of the perfect stage in Pyricularia from cereals and grasses. Ann Phytopathol Soc Jpn 42:511-515

Yaegashi H, Udagawa S (1978) The taxonomical identity of the perfect state of Pyricularia grisea and its allies. Can J Bot 56:180-183

Zhang N, Zhao S, Shen Q (2011) A six-gene phylogeny reveals the evolution of mode of infection in the rice blast fungus and allied species. Mycologia 103:1267-1276 